\title{
Transcranial Direct Current Stimulation (tDCS) Induces Adrenergic Receptor-Dependent Microglial Morphological Changes in Mice
}

\author{
Tsuneko Mishima, ${ }^{1}$ Terumi Nagai, ${ }^{1}$ Kazuko Yahagi, ${ }^{1}$ Sonam Akther, ${ }^{1,2,3}$ CYuki Oe, ${ }^{1}$ Hiromu Monai, ${ }^{1,4}$ \\ Shinichi Kohsaka, ${ }^{5}$ and ${ }^{-}$Hajime Hirase ${ }^{1,2,3}$
}

https://doi.org/10.1523/ENEURO.0204-19.2019

${ }^{1}$ Laboratory for Neuron-Glia Circuitry, RIKEN Center for Brain Science, Wako 351-0198, Japan, ${ }^{2}$ Brain and Body System Science Institute, Saitama University, Saitama 338-8570, Japan, ${ }^{3}$ Center for Translational Neuromedicine, Faculty of Health and Medical Sciences, University of Copenhagen, 2200 Copenhagen, Denmark, ${ }^{4}$ Faculty of Core Research Natural Science Division, Ochanomizu University, Tokyo 112-8610, Japan, and ${ }^{5}$ National Institute of Neuroscience, National Center of Neurology and Psychiatry, Kodaira 187-0031, Japan

\section{Visual Abstract}

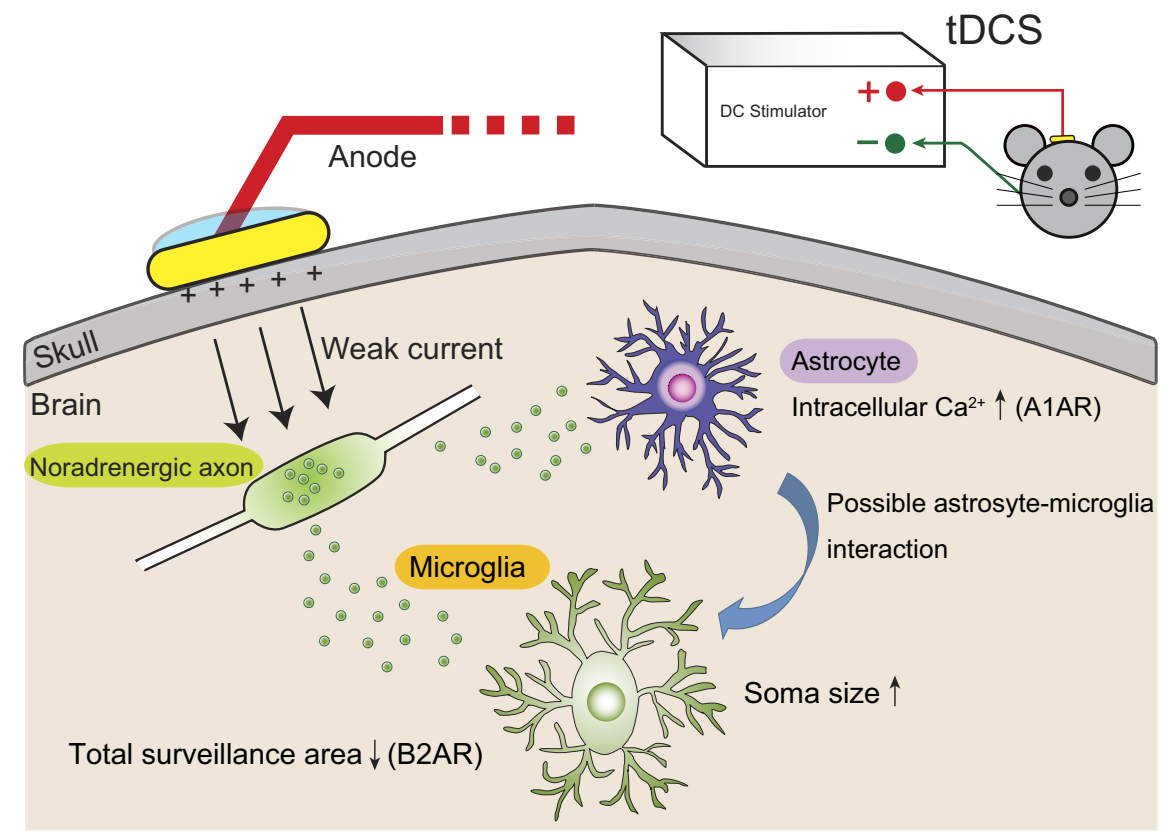

\section{Significance Statement}

Transcranial direct current stimulation (tDCS) is a neuromodulation procedure in which a weak electric direct current is delivered through the brain for tens of minutes. Despite reported positive effects, the mechanisms of tDCS stimulation are not yet well understood. Here, we examined microglial morphology in the mouse cortex after tDCS. We find that the morphology and morphologic dynamics of microglia are altered by tDCS in a manner dependent on adrenergic receptors, supporting the notion that (nor)adrenergic signaling is involved in tDCS. 
Transcranial direct current stimulation (tDCS) has been reported for its beneficial effects on memory formation and various brain disorders. While the electrophysiological readout of tDCS effects is subtle, astrocytes have been demonstrated to elicit $\mathrm{Ca}^{2+}$ elevations during tDCS in a rodent model. This study aimed to elucidate the effects of tDCS on another major glial cell type, microglia, by histology and in vivo imaging. tDCS performed in awake conditions induced a significant change in the pixel intensity distribution of Iba-1 immunohistochemistry, and microglial somata were enlarged when examined $3 \mathrm{~h}$ after tDCS. These effects were blocked by adrenergic receptor antagonists or in $\mathrm{IP}_{3} \mathrm{R} 2$ (inositol trisphosphate receptor type 2)-deficient mice, which lack large cytosolic $\mathrm{Ca}^{2+}$ elevations in astrocytes. No obvious changes were observed in isoflurane-anesthetized mice. Furthermore, in vivo two-photon imaging of microglia showed a reduction of motility that was blocked by a $\beta_{2}$-adrenergic receptor antagonist. Our observations add support for the influence of noradrenaline in tDCS and suggest possible interactions between microglia and astrocytes to express functional changes associated with tDCS.

Key words: iba1; in vivo ; microglia; norepinephrine; tDCS; two-photon imaging

\section{Introduction}

Noninvasive neuromodulation is a subject of intense research because of its potential for treating patients with neuropsychiatric and neurologic conditions. Transcranial direct current stimulation (tDCS) is the application of a constant and weak electric current to the brain through the skull. Typical parameters applied in humans are $1 \mathrm{~mA}$ over $\sim 30 \mathrm{~cm}^{2}$ for $10-30$ min (Bikson et al., 2016). A fair sized body of published literature suggests that tDCS has positive effects on cognitive abilities and could be an alternative treatment for various brain disorders (Fregni and Pascual-Leone, 2007; Nitsche et al., 2008, 2009; Brunoni et al., 2012; Dedoncker et al., 2016). On the other hand, there is a notable degree of skepticism due to mixed outcomes of tDCS experiments (Horvath et al., 2015a,b; Jalali et al., 2017; Medina and Cason, 2017; Kunzelmann et al., 2018; Turkakin et al., 2018). The skepticism has been, in part, strengthened by a recent study that suggested negligible tDCS-induced membrane potential changes in cerebral cortical neurons (Vöröslakos et al., 2018), implying limited involvement of neuronal discharge as the prevalent mechanism of tDCS.

The circuit and cellular mechanisms for tDCS remain to be understood. Glial cells represent electrically nonexcitable cells in the nervous system. They have been regarded as "support cells" for the normal function of neurons. Among glial cell types, astrocytes and microglia

Received May 29, 2019; accepted August 19, 2019; First published August 23 , 2019.

The authors declare no competing financial interests.

Author contributions: T.M., S.K., and H.H. designed research; T.M., T.N., K.Y., S.A., Y.O., and H.M. performed research; T.M., T.N., and K.Y. analyzed data; T.M. and H.H. wrote the paper.

This work was supported by the RIKEN Brain Science Institute and Center for Brain Science; KAKENHI Grants 16H01888, 18H05150, and 18K14859; and Human Frontier Science Program Grant RGP0036/2014.

Acknowledgments: We thank the members of the laboratory for comments on this manuscript. We also thank the RIKEN CBS-Olympus Collaboration Center for confocal imaging equipment and software, Keyence for fluorescence microscopy support, and Dr. Kazuhiro Sohya (National Center of Neurology and Psychiatry, Japan) for providing transgenic mice.

Correspondence should be addressed to Tsuneko Mishima at Tsuneko.mishima@riken.jp or Hajime Hirase at hirase@sund.ku.dk.

https://doi.org/10.1523/ENEURO.0204-19.2019

Copyright $\odot 2019$ Mishima et al.

This is an open-access article distributed under the terms of the Creative Commons Attribution 4.0 International license, which permits unrestricted use, distribution and reproduction in any medium provided that the original work is properly attributed. maintain the extracellular milieu by ion homeostasis and phagocytosis, respectively. Additionally, astrocytes and microglia have been reported to interact with neuronal synapses (Wake et al., 2013; Araque et al., 2014). We recently reported that astrocytic $\mathrm{Ca}^{2+}$ surges occur during tDCS in mice. Moreover, tDCS-induced astrocytic $\mathrm{Ca}^{2+}$ surges were shown to promote cortical plasticity and have beneficial effects in a mouse model of depression (Monai et al., 2016; Monai and Hirase, 2016, 2018). The recruitment of $\mathrm{Ca}^{2+}$ activities in astrocytes has prompted us to investigate another major glial cell type, microglia.

Microglia are sensitive to brain tissue damage and transform to reactive microglia on inflammation. Iba1 (ionized calcium binding adaptor molecule 1) immunohistochemistry $(\mathrm{IHC})$ visualizes the morphology of microglia, which is profoundly altered in reactive microglia. Following the published observation that reported the lack of pronounced microglial reactivity after tDCS (Monai et al., 2016), here we investigated Iba1 IHC in detail by digital image analysis. We report subtle, but significant effects of tDCS in an awake condition, but not under anesthesia, that depended on adrenergic receptors. Subsequently, we examined microglial motility by in vivo two-photon imaging and found that tDCS reduces microglial motility.

\section{Materials and Methods}

All animal procedures were performed in accordance with the RIKEN animal experimental committee regulations.

\section{Animals}

Adult C57BL/6J and $\mathrm{IP}_{3} \mathrm{R} 2$ (inositol trisphosphate receptor type 2) knock-out (KO) mice (Futatsugi et al., 2005) were used for immunohistochemical experiments (male, 2-4 months old). BAC-GLT1-G-CaMP7 line 817 mice (male, 2-5 months old; catalog \#G7NG817, RIKEN BioResource Research Center; resource ID: RBRC09650) were used for transcranial macroscopic imaging of neuronal and astrocytic $\mathrm{Ca}^{2+}$ activity (Monai et al., 2016). Iba1-GFP mice (male, 3-10 months old; Hirasawa et al., 2005) were used for in vivo two-photon imaging of microglial morphology.

\section{Surgical procedures}

Mice were deeply anesthetized with isoflurane (1.5$2.0 \%$ ), and their scalps were exposed by shaving. Each 
A

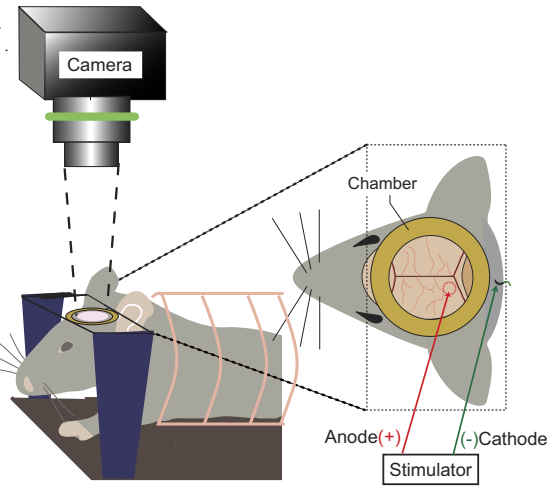

C

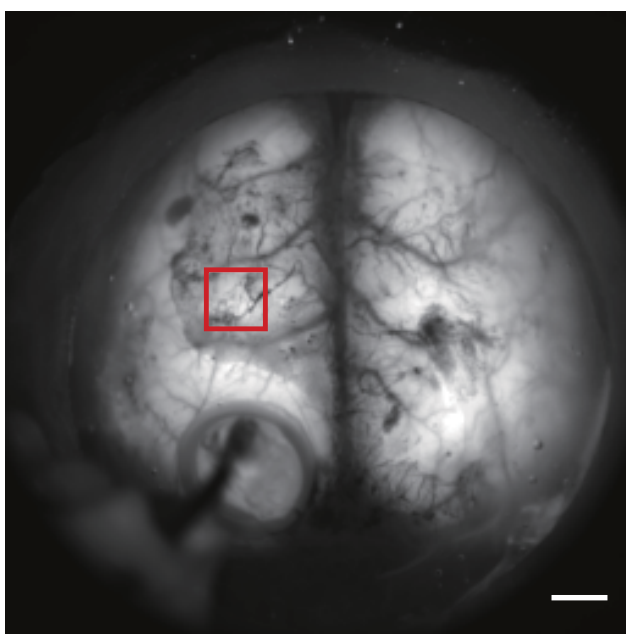

B

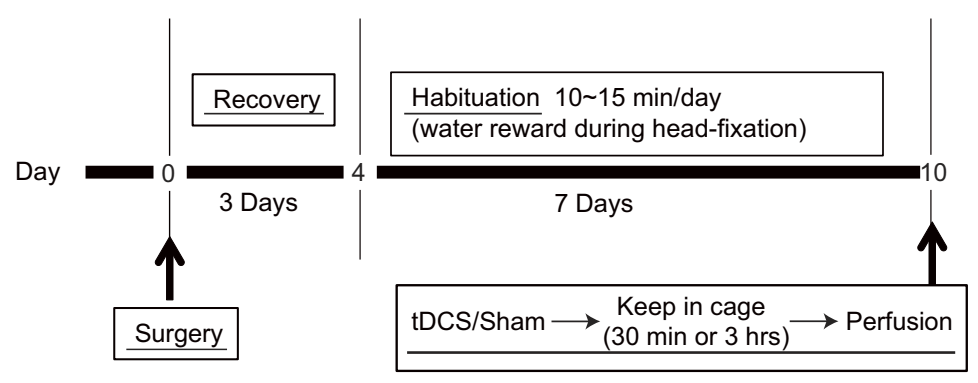

Figure 1. Head-restraint tDCS experiment. $\boldsymbol{A}$, Experimental setup for tDCS. $\boldsymbol{B}$, Experimental schedule of immunohistochemical experiment. C, Top view of a BAC-GLT1-G7 Line 817 (G7NG817) mouse. Fluorescent $\mathrm{Ca}^{2+}$ signal is transcranially observable. Signals $\sim 3 \mathrm{~mm}$ anterior to the anodal site $\left(1 \times 1 \mathrm{~mm}^{2}\right.$ red square) are plotted from four mice (right, top traces). The bold trace on the bottom is the mean of the four traces, and the shaded areas represent SE. The red arrowhead and line indicate the onset of tDCS. Scale bar, $1 \mathrm{~mm}$. tDCS-induced $\mathrm{Ca}^{2+}$ elevations were not observed in isoflurane-anesthetized mice (Extended Data Fig. 1-1).

mouse was fixed on a stereotaxic apparatus (Narishige) under isoflurane anesthesia. Throughout the surgery and experiments with anesthetized mice, the body temperature was kept at $37^{\circ} \mathrm{C}$ with a heating blanket (BWT-100A, Bio Research Center). After topical application of xylocaine ointment (2\% lidocaine) on the scalp, the skull above the sensory cortex was exposed by incision of the scalp and temporal muscle. A custom-made chamber ring was glued to the skull with cyanoacrylate superglue. After the glue settled, we applied dental cement (Fuji LUTE BC, GC Corporation; Super-Bond C\&B, Sun Medical) for reinforcement. For two-photon imaging, the inner cavity of the chamber ring was reinforced with additional dental cement to secure the interface for an objective lens. Once the chamber ring was rigidly attached, the mouse was fixed on a custom-made stage via the chamber ring. Thereafter, a small craniotomy $(\varphi=3 \mathrm{~mm}$, with intact dura) was carefully made using a dental drill.

\section{Habituation to head restraint}

The postsurgical recovery period was at least $3 \mathrm{~d}$ for IHC experiments and 2 weeks for in vivo two-photon imaging experiments. Following the recovery period, mice were placed on a water restriction schedule and subjected to an acclimatization procedure for head restraint (Fig. 1B). Food was given ad libitum. The acclimatization procedure was performed for $7 \mathrm{~d}$.

On day 1, each mouse was held in the experimenter's hands and water was given via a syringe $(\sim 0.2 \mathrm{ml})$. During handling, we let the mouse explore until it entered into a body tube similar to the one used with the tDCS apparatus. If the mouse entered the body tube, we repeated the procedure four to five times. The total handling time was 10 min for each mouse. From day 2, the mouse continued to be acclimatized to the experimenter and apparatus with a water reward $(0.1-0.2 \mathrm{ml})$ for each entry to the body tube. At this point, the head of the mouse was quickly $(<10-20 \mathrm{~s})$ fixed to the apparatus via the chamber ring with its body in the tube. Additional water and sunflower seeds were provided during head fixation (10-15 $\mathrm{min}$ ). The total amount of water given during head fixation was $1.0 \mathrm{ml} / \mathrm{d}$. In some mice, for in vivo two-photon imaging acclimatization was performed for $>7 \mathrm{~d}$. 


\section{Transcranial DC stimulation}

tDCS was applied on mice under anesthesia (2\% isoflurane) or in awake conditions. In either condition, the anode (stainless wire, $\varphi=350 \mu \mathrm{m}$ ) was placed on a sodium chloride-based conductive gel interface (Z101BA, NIHON-KODEN) spread over a circular area $(\varphi=\sim 2 \mathrm{~mm})$ above the primary visual cortex (anteroposterior, -2.9 $\mathrm{mm}$; mediolateral $2.0 \mathrm{~mm}$ ). The cathode was connected to the neck skin after topical application of xylocaine ointment. DC (0.1 mA, $10 \mathrm{~min})$ was applied with a custommade isolated constant-current supply.

\section{Histology}

After tDCS application, mice were kept for 30 min or 3 $\mathrm{h}$ before they were killed. After deep anesthesia by urethane, they were first perfused with $0.9 \% \mathrm{NaCl}$ and later with fixative solution (4.0\% paraformaldehyde in $0.1 \mathrm{M}$ phosphate buffer, $\mathrm{pH}$ 7.4). Following brain removal and overnight postfixation in the same fixative, coronal slices $(60 \mu \mathrm{m})$ were prepared using a microslicer (PRO 7, Dosaka). For Iba1 staining, sections were incubated in a buffer containing the primary antibody (Tris-buffered saline with $0.1 \%$ Triton X-1000; 1:2000; catalog \#01919741, Wako) overnight. The sections were subsequently washed in PBS and incubated with the Cy3-conjugated secondary antibody (Invitrogen) for $2 \mathrm{~h}$ for fluorescent labeling. To evaluate DSP4 [N-(2-chloroethyl)- $N$-ethyl-2bromobenzylamine hydrochloride] efficacy, noradrenergic fibers were labeled by anti-tyrosine hydroxylase $(\mathrm{TH})$ antibody (1:1000; catalog \#AB152, Millipore) using sagittal slices $(60 \mu \mathrm{m})$. For positive control of microglial reactivity, Escherichia coli lipopolysaccharide (LPS; $0.5 \mathrm{mg} / \mathrm{kg}$ ) was administered by intraperitoneal injection $2 \mathrm{~d}$ before the mice were killed.

\section{Confocal imaging}

Immunolabeled cortical microglia (V2 area) were examined using a confocal microscope (FV1000, Olympus). Images were acquired with a $60 \times$ water-immersion objective (UPlanSApo; numerical aperture, 1.20) at an excitation wavelength of $559 \mathrm{~nm}$. Imaged areas covered $211.761 \times 211.761 \mu \mathrm{m}^{2}(1024 \times 1024$ pixels $)$ with an optical sectioning of $0.5 \mu \mathrm{m}$. Images were scanned with the one-way mode ( $8 \mu \mathrm{s} /$ pixel exposure).

\section{Drug administration}

In some experiments, the following drugs were administered before tDCS by intraperitoneal injection: ICI81551 ( $5 \mathrm{mg} / \mathrm{kg}$ body weight, $30 \mathrm{~min}$ before; Tocris); and Prazosin $(10 \mathrm{mg} / \mathrm{kg}, 30 \mathrm{~min}$ before; Sigma-Aldrich). For the ablation of noradrenergic neurons, DSP4 (Sigma-Aldrich) was injected 11 and $7 \mathrm{~d}$ before tDCS application (50 $\mathrm{mg} / \mathrm{kg}$, i.p., each time). Drugs were dissolved in $0.9 \%$ $\mathrm{NaCl}$.

\section{In vivo imaging of microglial morphology}

Adult Iba1-EGFP transgenic mice (Hirasawa et al., 2005), in which EGFP is expressed exclusively in microglia, were used to monitor microglial morphologic dynamics. All mice were habituated to the experimental apparatus for $>7 \mathrm{~d}$. On the day of imaging, the mouse was set on a custom-made stage under a two-photon microscope (B-Scope, Thorlabs). Microglia located $>50$ $\mu \mathrm{m}$ below the pial surface were imaged under awake conditions at a wavelength of $920 \mathrm{~nm}$. The laser power was adjusted to $\sim 12 \mathrm{~mW}$ at the preparation (Hines et al., 2009; Wake et al., 2009; Pfeiffer et al., 2016). Depth stacks $(24-26$ slices, $2 \mu \mathrm{m} \mathrm{z}$ interval, $512 \times 512$ pixels corresponding to $101 \times 101 \mu \mathrm{m}^{2}$ or $201 \times 201 \mu \mathrm{m}^{2}$ ) were acquired every $60 \mathrm{~s}$.

\section{Analysis}

Iba1 IHC image analysis

Confocal images were used for pixel intensity analysis. Image stacks extending to $15 \mu \mathrm{m}$ thickness were collapsed into 2D images by maximum intensity projection. Pixel intensities were converted to $z$ scores, and the cumulative distribution was computed for each collapsed 2D image.

For soma size analysis, confocal image stacks (45-50 $\mu \mathrm{m}$ thickness) were first filtered with a $3 \times 3 \times 3$ median filter. The resultant image stacks were collapsed into $2 \mathrm{D}$ images by maximum intensity projection. To correct for uneven background, the rolling ball method with a radius of 30 pixels was used for background subtraction. Thereafter, the images were subjected to a $3 \times 32 \mathrm{D}$ median filter followed by binarization with Yen's thresholding method (ImageJ, National Institutes of Health) for soma extraction. In some cases, manual adjustments of threshold were needed. Extracted somata were approximated to ellipses. Following these automated procedures in ImageJ, extracted somata were validated by manual inspection. The median of microglial soma size distribution from each mouse was taken as a data point for statistical comparisons.

\section{TH image analysis}

The efficacy of DSP4 was evaluated by calculating the mean intensity of posterior cortical layer $1 \mathrm{TH}$-positive $\left(\mathrm{TH}^{+}\right)$innervation using Image J. Briefly, sagittal brain section images ( $60 \mu \mathrm{m}$ thickness) were acquired by a Keyence microscope (BZ-X710; $0.37 \mu \mathrm{m}$ pixel size). Ten to 12 contiguous regions of interest (ROls; $100 \times 100 \mu \mathrm{m}$ each) were allocated to occupy layer 1 . A background intensity value was calculated from a neighboring parenchymal area that does not contain $\mathrm{TH}^{+}$axons. The mean $\mathrm{TH}^{+}$ signal intensity of each $\mathrm{ROI}$ was computed as the mean pixel intensity minus the background intensity.

\section{Microglial motility assessment}

Quantification of microglial surveillance was performed using custom-written ImageJ and MATLAB programs (MathWorks). The maximum intensity projection image was computed for each time point of xyzt image stack. The resultant $x y t$ image stack was registered for $x y$ motion correction. Next, each slice of the xyt stack was processed by the ImageJ "Subtract Background" plugin to subtract smooth continuous background with a ball size of 30 pixels. Thereafter, images were treated with a 2D 3 $\times 3$ median filter. After this preprocessing, rectangular areas containing the morphologic extent of single microglia were extracted as separate image stacks. These cellwise image stacks were then binarized with a single 
Table 1: Statistical table

\begin{tabular}{|c|c|c|c|c|}
\hline & Sample number: cells (animals) & Test type & $p$ Value & Power \\
\hline a & Sham: 334(7), Ctl: 315(7) & Mann-Whitney Wilcoxon rank sum test & 0.1 & \\
\hline $\mathrm{b}$ & Sham: $315(7)$, tDCS: $314(7)$ & Mann-Whitney Wilcoxon rank sum test & 0.16 & \\
\hline c & Sham: 309(7), tDCS: 301(7) & Mann-Whitney Wilcoxon rank sum test & $* * 0.017$ & \\
\hline$d$ & Sham: 278(6), tDCS: 296(7) & Mann-Whitney Wilcoxon rank sum test & 0.95 & \\
\hline e & Sham: $285(7)$, tDCS: $319(7)$ & Mann-Whitney Wilcoxon rank sum test & 0.073 & \\
\hline f & Sham: 238(6), tDCS: 356(7) & Mann-Whitney Wilcoxon rank sum test & 0.73 & \\
\hline g & Sham: $274(7)$, tDCS: $310(7)$ & Mann-Whitney Wilcoxon rank sum test & 0.8 & \\
\hline $\mathrm{h}$ & Sham: $266(6)$, tDCS: $282(6)$ & Mann-Whitney Wilcoxon rank sum test & 0.48 & \\
\hline & Sham: 13(8) & Paired $t$ test & 0.82 & 0.055 \\
\hline & tDCS: 11(8) & Paired $t$ test & $* * 0.014$ & 0.77 \\
\hline $\mathrm{k}$ & Sham: 13(8), tDCS: 11(8) & Mann-Whitney Wilcoxon rank sum test & $* * * 0.006$ & \\
\hline 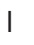 & Sham: 11(3), tDCS: $9(2)$ & Mann-Whitney Wilcoxon rank sum test & $* * 0.015$ & \\
\hline $\mathrm{m}$ & Sham: 9(3), tDCS: $12(3)$ & Mann-Whitney Wilcoxon rank sum test & $* * 0.023$ & \\
\hline
\end{tabular}

$* p<0.05, * * p<0.03, * * * p<0.01$

threshold determined by Li's Minimum Cross Entropy method (ImageJ). Noise reduction was then performed by a cycle of erosion and dilation. The normalized surveillance area at time $t$ was calculated as the number of pixels that were occupied by the microglia at least once since the beginning of imaging until a given time $t$, divided by the number of pixels occupied by the microglia at the beginning. Normalized surveillance area is therefore a monotonically increasing function of time (see Fig. $6 C, D$, example). The surveillance index is defined as the ratio of normalized surveillance areas of a microglia in two different sessions [e.g., control ("Before") vs post-tDCS ("After")].

\section{Statistical analyses}

Statistical analyses were performed using lgor Pro (WaveMetrics). Student's paired $t$ tests and WilcoxonMann-Whitney rank sum tests were used for the comparison of two sample populations with matched data and unmatched data, respectively, unless otherwise noted. Data are expressed as the mean \pm SEM, and $p$ values $<0.05$ were considered statistically significant. Statistical values are reported in Table 1.

\section{Results}

First, we confirmed tDCS-induced cortex-wide $\mathrm{Ca}^{2+}$ elevations (Monai et al., 2016) in the present setting using G7NG817 transgenic mice that express the G-CaMP7 $\mathrm{Ca}^{2+}$ sensor in astrocytes and a subpopulation of neurons. Mice had been acclimatized to be rigidly fixed to a head-restraint platform, where tDCS $(0.1 \mathrm{~mA}, 10 \mathrm{~min})$ and transcranial fluorescence imaging were performed (Fig. $1 A, B$; see Materials and Methods). Cortical $\mathrm{Ca}^{2+}$ signals elevated immediately after the passage of the $D C$ current. The peak amplitude of the G-CaMP7 response measured $\sim 3 \mathrm{~mm}$ anterior to the anodal position was $39.7 \pm 4.1 \%$ (Fig. $1 C ; N=4$ mice), showing that tDCS-induced $\mathrm{Ca}^{2+}$ elevation is observable with the head chamber-ring configuration. Notably, tDCS-induced $\mathrm{Ca}^{2+}$ elevations were not observed in isoflurane-anesthetized mice (Extended Data Fig. 1-1). Having demonstrated the effectiveness of tDCS, we used C57BL/6 mice to investigate microglial morphology after tDCS by lba1 IHC. Mice were killed either 30 min or $3 \mathrm{~h}$ after tDCS for perfusion fixation.

\section{Iba1 IHC patterns are affected by tDCS in awake mice}

Iba1 IHC visualized highly ramified microglial morphology throughout brain slices of sham-operated, LPStreated, and tDCS mice (Fig. $2 A, B, I, J)$. To investigate the impact of tDCS on the wide-field appearance of Iba1 IHC, we computed the pixel intensity distribution, which is a proxy of global morphologic changes. We analyzed layer 2 and 3 of the visual cortex located below the anode, since a previous study demonstrated that tDCS-mediated plasticity occurs in these layers (Monai et al., 2016). Pixel intensities were converted to $z$ scores with which the cumulative distributions were plotted. We compared head ring-implanted, unrestrained control mice (Ctl group) with head ring-implanted, acclimatized, $25 \mathrm{~min}$ headrestrained mice (Sham group) to evaluate the possible effects of head restraint. In Figure 2C, we demonstrate that cumulative pixel intensity distribution is similar between the $\mathrm{Ctl}$ and Sham groups, whereas the pixel intensity distribution shifted significantly in mice with reactive microglia caused by LPS. These results suggest that the head-restraining procedure in acclimatized mice does not cause reactivity in cortical microglia.

Next, we compared tDCS and sham-treated mice. The combination of two conditions [isoflurane-anesthetized (isofl) or awake] and two time points (30 min and $3 \mathrm{~h}$ after tDCS) were investigated (Fig. 2D-G). Pixel intensity distribution was similar between sham and tDCS for isofl 30 min, isofl $3 \mathrm{~h}$, and awake 30 min experiments; however, the awake $3 \mathrm{~h}$ tDCS data exhibited a visible deviation from the sham group (Fig. 2G). This deviation was caused by a higher proportion of pixels at the high-intensity end. For instance, tDCS had a relatively large presence of pixels that had a $z$ score $>0.6(21.2 \pm 1.6 \%$ vs $18.2 \pm 1.4 \% ; p$ $=1.7 \mathrm{e}-5, t$ test). Moreover, a high-intensity cluster that has a $z$ score $>2$ was apparent in the pixel intensity histogram (Fig. 2H). Consistent with this observation, thresholding with $z>2$ preserved more microglial structures in awake $3 \mathrm{~h}$ tDCS images than the sham counterpart (Fig. 2l,J). While the cumulative pixel intensity histogram of awake $3 \mathrm{~h}$ tDCS deviated in the same direction as LPS, microglial morphology appeared normal with fine ramified processes throughout the extent of the cor- 
A Sham

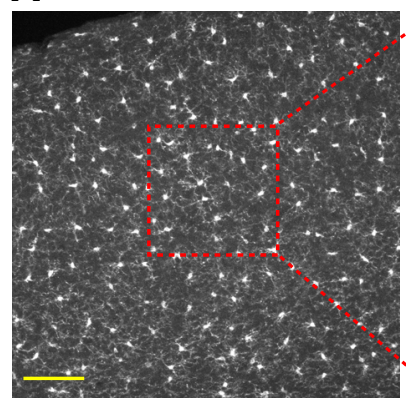

C

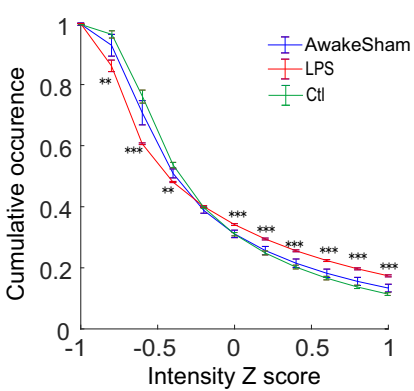

F

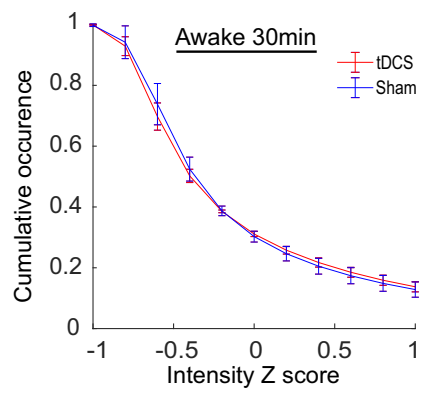

I Sham

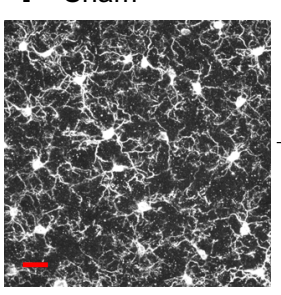

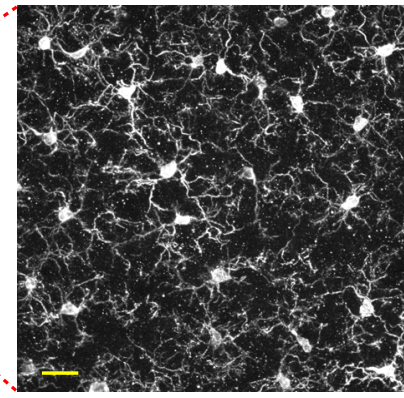

D

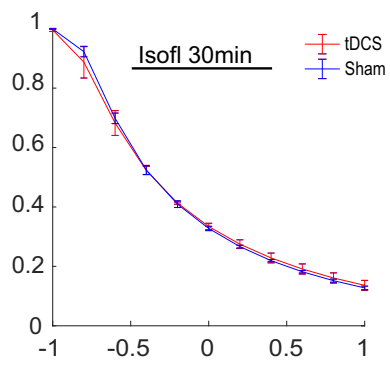

G

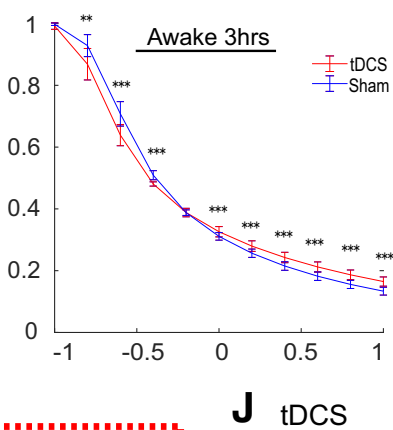

J tDCs
B LPS

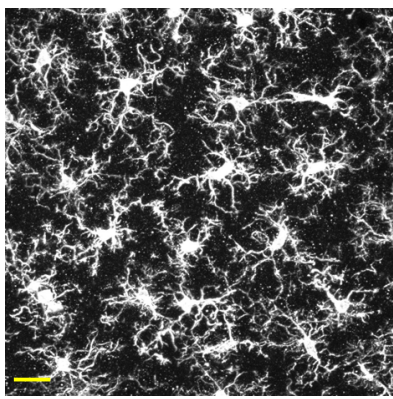

E

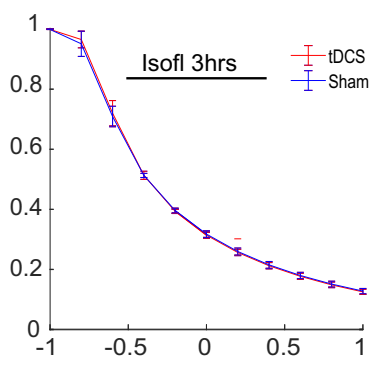

H

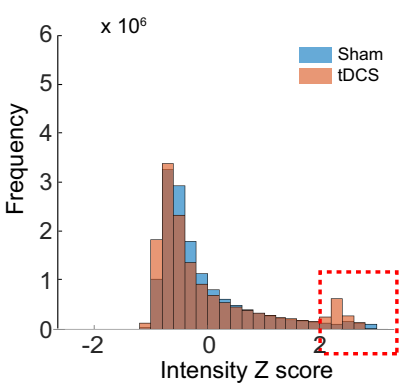

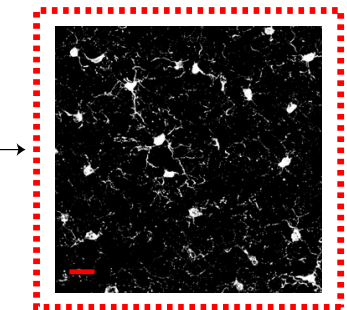
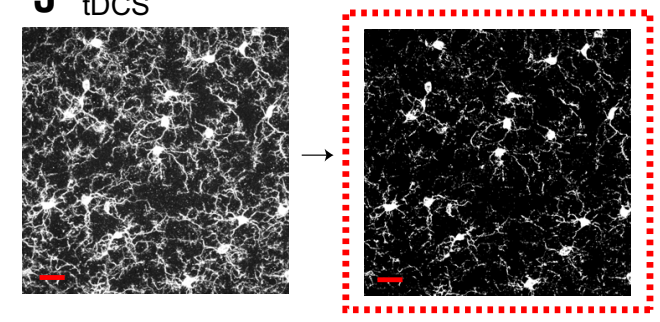

Figure 2. Intensity analysis of microglial confocal images. $\boldsymbol{A}, \boldsymbol{B}$, Representative images of Cy3-labeled Iba1 IHC by maximum intensity projection obtained in Sham- and LPS-treated mice. Yellow scale bars: $\boldsymbol{A}$, left, $100 \mu \mathrm{m} ; \boldsymbol{A}$, right, $\boldsymbol{B}, 20 \mu \mathrm{m}$. $\boldsymbol{C}$, Cumulative pixel intensity distributions from unrestrained $\mathrm{Ctl}$ and head-restrained Sham groups were similar and distinct from the LPS-treated group. $\boldsymbol{D}-\boldsymbol{G}$, Intensity was compared between tDCS- and Sham-treated groups under the isoflurane-anesthetized $(\boldsymbol{D}, \boldsymbol{E})$ or awake $(\boldsymbol{F}, \boldsymbol{G})$ conditions, perfused at $30 \mathrm{~min}$ or $3 \mathrm{~h}$ after sham/tDCS. $\boldsymbol{H}$, In awake mice, the pixel intensity histogram indicates that there is a cluster at $z$ score $>2$ (i.e., mean +2 SDs) region in the tDCS group (dotted red square). $\boldsymbol{I}, \boldsymbol{J}$, Representative images from a sham-treated mouse and a tDCS-treated mouse. Images in the red squares correspond to the thresholded images on the left at the mean +2 SDs. Red scale bars, $20 \mu \mathrm{m}$. $* * p<0.01, * * * p<0.001$.

tex in all tDCS experiments. Thus, tDCS does not appear to cause inflammatory responses.

\section{tDCS enlarges microglial somata in awake mice}

While the $z$ score-based pixel intensity distribution analysis detected changes in the global appearance of images, it falls short of providing information on specific aspects of morphologic alterations. Microglial soma size has been reported to be sensitive to brain environmental changes (Kongsui et al., 2015). Therefore, we measured microglial soma size from Iba1 IHC images (Fig. $3 A-C$; see Materials and Methods). First, we compared the median microglial soma size of individual animals (43 cells per mouse on average) for unrestrained control and headrestrained sham groups as we did in Figure $2 C$. Figure $3 D$ indicates that microglia soma sizes are similar between 
A

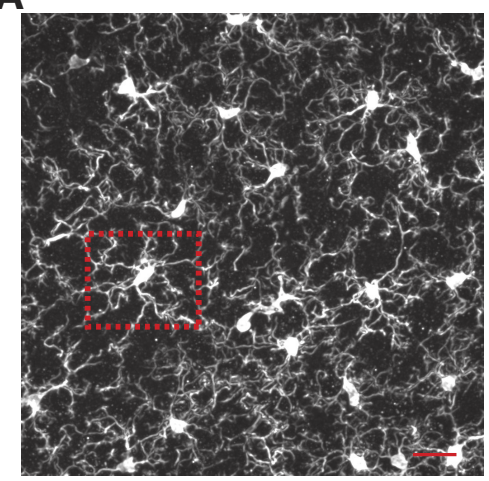

C

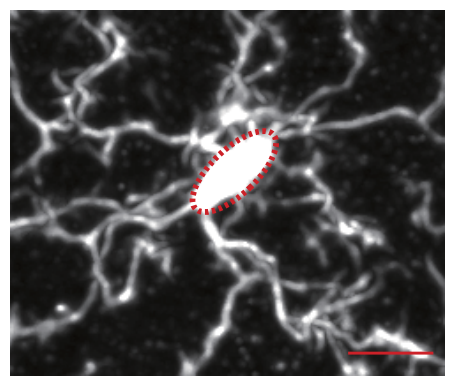

E

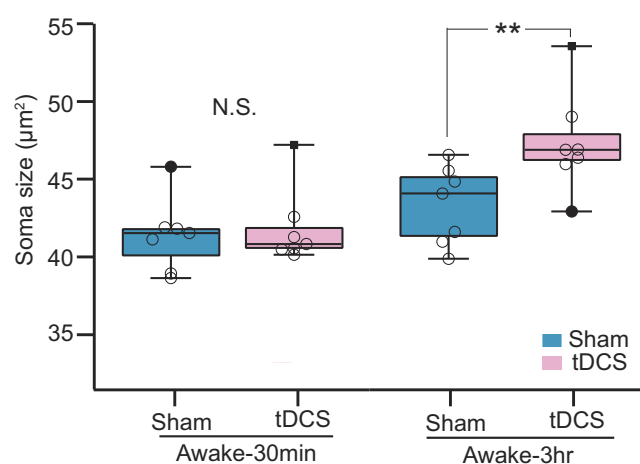

B

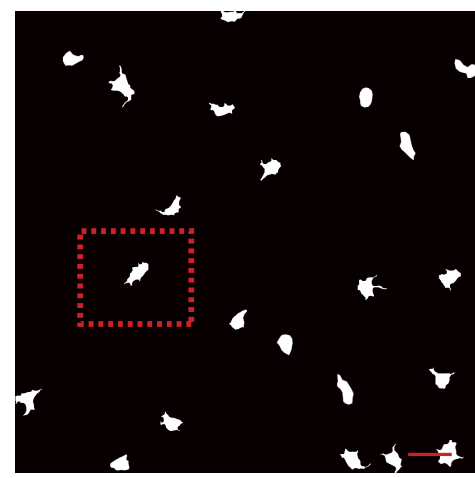

D

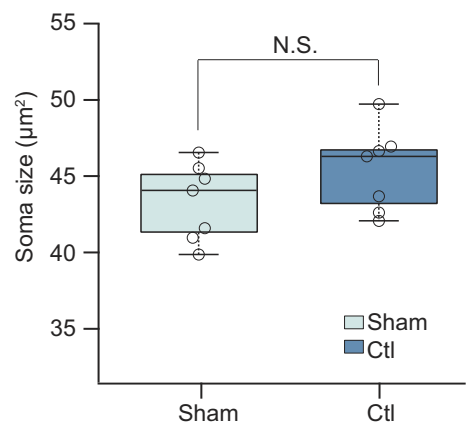

F

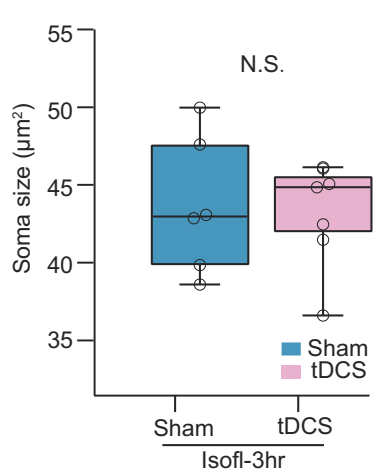

Figure 3. Quantification and comparison of microglial soma size. A, Example image of an Iba1 IHC confocal image stack collapsed by maximum intensity projection. Scale bar, $20 \mu \mathrm{m}$. $\boldsymbol{B}$, Digitally processed image of $\boldsymbol{A}$ for soma extraction. $\boldsymbol{C}$, Example of the elliptic approximation of soma $(\boldsymbol{A}, \boldsymbol{B}$, red dotted square). $\boldsymbol{D}$, Comparison of median values of microglial soma areas between Shamstimulated and unrestrained control mice ( $p=0.1^{\mathrm{a}}$, Mann-Whitney Wilcoxon rank sum test). Scale bars: $\boldsymbol{A}, \boldsymbol{B}, 10 \mu \mathrm{m} ; \boldsymbol{C}, 20 \mu \mathrm{m}$. $\boldsymbol{E}$, Comparison of microglial soma size in awake mice with/without tDCS treatment at different time points ( $30 \mathrm{~min}$ or $3 \mathrm{~h})$ after tDCS. Microglial soma size was larger in the tDCS group in the awake $3 \mathrm{~h}$ experiment ( $p=0.017^{\mathrm{c}}$, Mann-Whitney Wilcoxon rank sum test). Each group contains seven mice. $\boldsymbol{F}$, Microglial soma size comparison in isoflurane-anesthetized mice (Isofl- $3 \mathrm{hr}) . * * p<0.03, \mathrm{~N} . \mathrm{S}$. not significant.

the control and sham groups (Ctl group: $45.4 \pm 1.0 \mu \mathrm{m}^{2}$, 7 mice; Sham group: $43.4 \pm 1.0 \mu \mathrm{m}^{2}, 7$ mice; $p=0.16^{\mathrm{b}}$, Mann-Whitney Wilcoxon test), suggesting that the microglial soma size of the Sham group serves as a valid control for tDCS experiments.

Soma size did not differ significantly between the awake 30 min tDCS and Sham groups (Sham group: 41.5 $\pm 0.92 \mu \mathrm{m}^{2}, 7$ mice; tDCS group: $41.9 \pm 0.9 \mu \mathrm{m}^{2}, 7$ mice; Fig. $\left.3 E ; p=1.0^{\mathrm{a}}\right)$. In awake $3 \mathrm{~h}$ experiments, soma size was significantly larger in the tDCS group $\left(p=0.017^{\mathrm{e}}\right.$; Sham group: $43.4 \pm 1.0 \mu \mathrm{m}^{2}, 7$ mice; tDCS group: $47.5 \pm$ $1.2 \mu \mathrm{m}^{2}, 7$ mice; Fig. $3 E$ ). On the other hand, soma size did not differ significantly when tDCS was performed on isoflurane-anesthetized mice ( 3 h group: $p=0.95^{f}$, Sham group: $43.7 \pm 1.8 \mu \mathrm{m}^{2}, 6$ mice; vs tDCS group: $43.2 \pm 1.3$ $\mu \mathrm{m}^{2}, 7$ mice; Fig. 3F). These results were consistent with the pixel intensity distribution analysis (Fig. 2) and suggest that isoflurane anesthesia hampers tDCS-induced microglial soma enlargement.

\section{tDCS-induced microglial soma enlargement is dependent on adrenergic receptors}

Recent human and animal studies have implicated the involvement of noradrenaline in tDCS (Monai et al., 2016; 
A

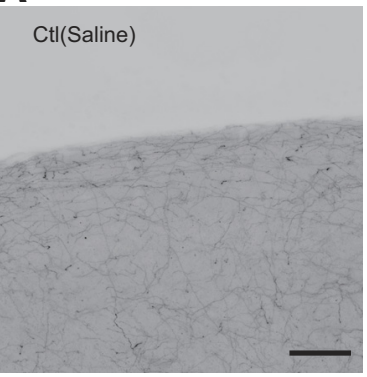

B

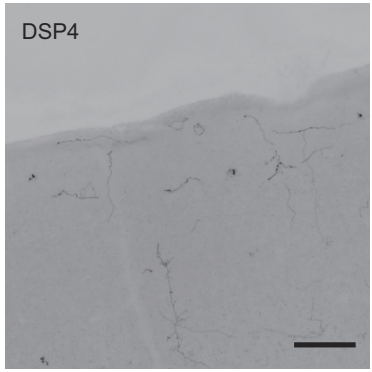

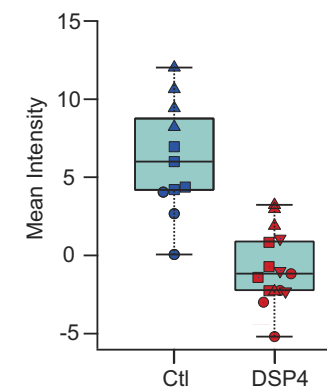

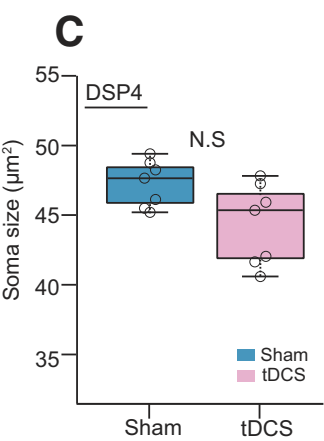

Figure 4. tDCS-induced microglial somatic enlargement depends on noradrenaline. $\boldsymbol{A}$, Example of cortical image (inverted grayscale) from saline- (left) or DSP4- (right) pretreated mice stained with $\mathrm{TH}$ antibody. $\boldsymbol{B}$, Mean intensity analysis of $\mathrm{TH}^{+}$fiber. Each group contains data from three mice. Data from the same animals are plotted with the same symbol and color. Scale bars, $100 \mu \mathrm{m}$. $\boldsymbol{C}$, Comparison between median glial soma size from sham- and tDCS-treated mice (Sham group: 7 mice; tDCS: 7 mice; $p=0.073^{\mathrm{e}}$, Mann-Whitney Wilcoxon rank sum test). N.S. not significant.

Kuo et al., 2017; Monai and Hirase, 2018; Souza et al., 2018). To examine the possible contribution of noradrenaline to tDCS-induced microglia soma size, we ablated noradrenergic cells in the locus ceruleus using the neurotoxin DSP4 (Bekar et al., 2008), which was confirmed by $\mathrm{TH}$ staining in the sensory cortex (Fig. $4 A, B$ ). Following noradrenergic neuron ablation, we performed tDCS using the awake $3 \mathrm{~h}$ protocol. As a result, DSP4-treated mice did not show a microglial soma enlargement after tDCS (Fig. 4C; Sham group: $47.3 \pm 0.6 \mu \mathrm{m}^{2}, 7$ mice; tDCS group: $44.5 \pm 1.1 \mu \mathrm{m}^{2}, 7$ mice; $p=0.073^{\mathrm{e}}$ ).

Since astrocytes exhibit profound $\alpha_{1}$-adrenergic receptor (A1AR)-mediated $\mathrm{Ca}^{2+}$ elevations by tDCS (Monai et al., 2016), astrocytic $\mathrm{Ca}^{2+}$ signaling possibly plays a role in the microglial soma enlargement via an intercellular communication. To examine this possibility, we used $\mathrm{IP}_{3} \mathrm{R} 2$ knock-out mice in which $\mathrm{G}_{\mathrm{q}}$ GPCR (e.g., A1AR)activated intracellular $\mathrm{Ca}^{2+}$ elevation is diminished in astrocytes. Awake $3 \mathrm{~h}$ tDCS did not result in significant microglial soma size changes in $\mathrm{IP}_{3} \mathrm{R} 2 \mathrm{KO}$ mice $\left(p=0.73^{\mathrm{f}}\right.$; Sham group: $44.6 \pm 1.4 \mu \mathrm{m}^{2}, 6$ mice; tDCS group: $44.4 \pm$ $0.56 \mu \mathrm{m}^{2}, 7$ mice; Fig. $5 A$ ). We next examined the involvement of A1AR using the specific antagonist prazosin in wild-type C57BL/6J mice. Similar to $\mathrm{IP}_{3} R 2 \mathrm{KO}$ mice, prazosin-treated mice did not display tDCS-induced mi- croglial soma enlargement compared with the Sham control group that also received the antagonist pretreatment $\left(p=0.8 \times g\right.$; Sham group: $42.6 \pm 0.9 \mu \mathrm{m}^{2}, 7$ mice; tDCS group: $42.3 \pm 0.7 \mu \mathrm{m}^{2}, 7$ mice; Fig. $5 B$ ). These results suggest that tDCS-triggered noradrenaline release affects microglial soma enlargement via A1AR activation and the downstream astrocytic $\mathrm{IP}_{3} \mathrm{R} 2$-dependent $\mathrm{Ca}^{2+}$ signaling pathway.

Furthermore, we asked whether activation of $\beta$-adrenergic receptors is also involved. In particular, microglia are known for high levels of $\beta_{2}$-adrenergic receptor (B2AR) expression (Tanaka et al., 2002, Gyoneva and Traynelis, 2013). Accordingly, mice were pretreated with ICl181551, a selective B2AR blocker, and soma sizes were compared. In the ICl181551 group, tDCS-induced soma size enlargement was not observed $\left(p=0.48^{\mathrm{h}}\right.$; Sham group: $45.9 \pm 0.8 \mu \mathrm{m}^{2}, 6$ mice; tDCS group: $45.2 \pm$ $1.7 \mu \mathrm{m}^{2}, 6$ mice; Fig. $5 C$ ). These results are indicative of noradrenergic involvement in tDCS-induced microglial changes and suggest that both A1ARs and B2BRs are involved in tDCS-induced microglial soma enlargement.

\section{tDCS decreases microglial surveillance area in vivo}

One of the striking features of microglia is the motility of their ramified processes. Here, we directly examined the
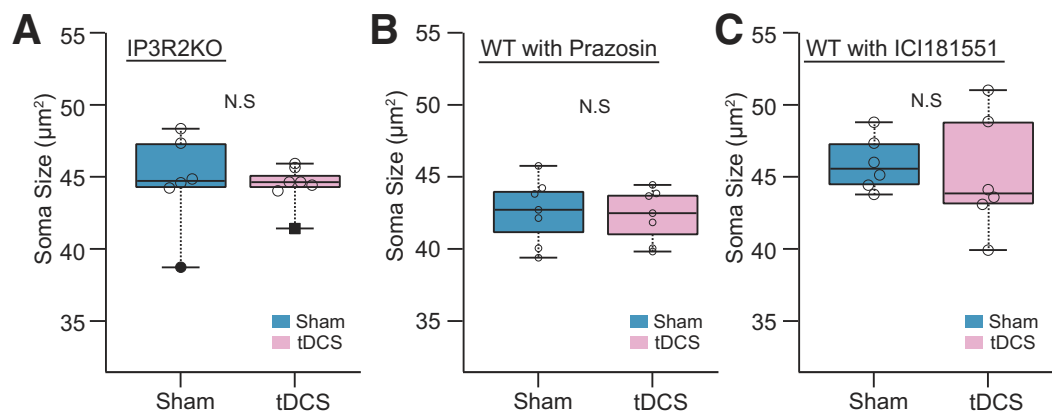

Figure 5. tDCS-induced microglial somatic enlargement depends on B2AR and A1AR pathways. $\boldsymbol{A}$, Comparison between median microglial soma size between Sham- and tDCS-treated $\mathrm{IP}_{3} \mathrm{R} 2 \mathrm{KO}$ mice (Sham group: 6 mice; tDCS group: 7 mice; $p=0.73^{f}$ ). $\boldsymbol{B}, \boldsymbol{C}$, Comparison of microglial soma size between Sham- and tDCS-treated wild-type strain C57BL/6J with prazosin (B; Sham group: 7 mice; tDCS group: 7 mice; $p=0.8 \times g$ ), or ICl181551 pretreatment (C; Sham group: 6 mice; tDCS group: 6 mice; $\left.p=0.48^{\mathrm{h}}\right)$. N.S. not significant. 
A

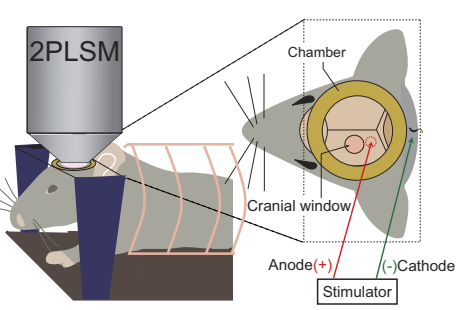

C

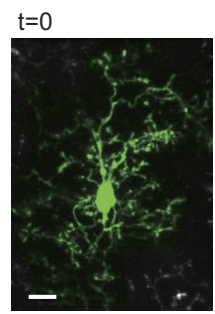

E

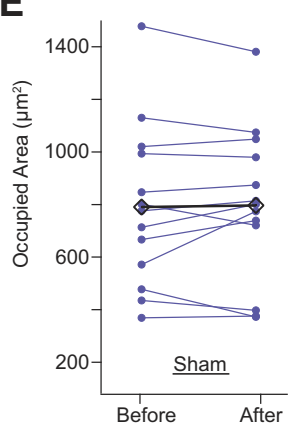

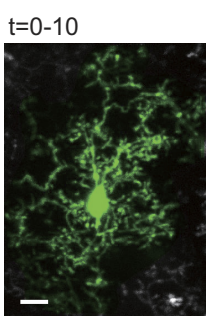

F

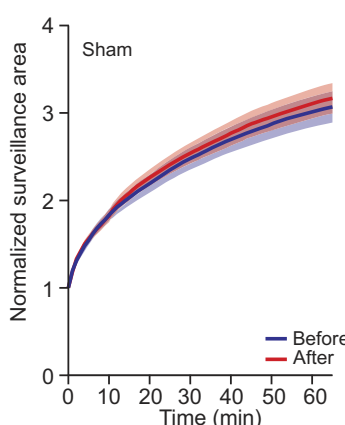

B

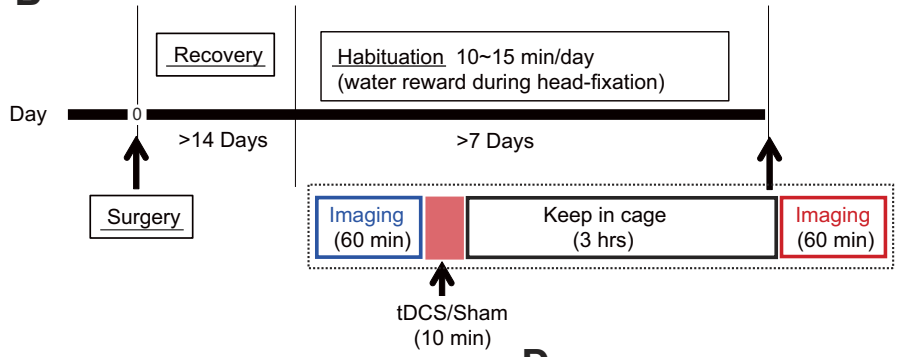

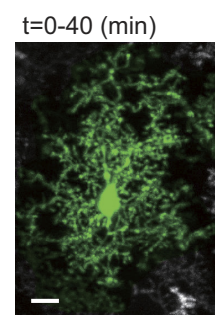

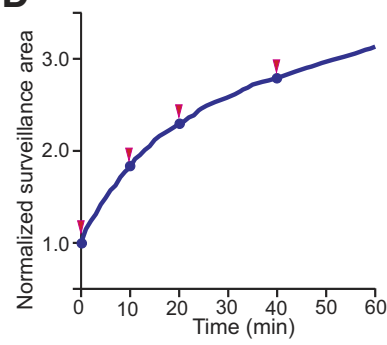

G

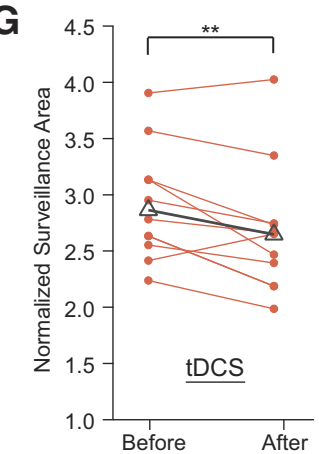

Figure 6. In vivo monitoring of microglial morphologic dynamics. $\boldsymbol{A}, \boldsymbol{B}$, Experimental setup $(\boldsymbol{A})$ and time schedule of in vivo two-photon imaging $(\boldsymbol{B})$. $\boldsymbol{C}$, Representative images of a microglia overlaid from $t=0$ to respective time points $(10,30$, and $40 \mathrm{~min})$. $\boldsymbol{D}$, Normalized surveillance area curve during $60 \mathrm{~min}$ imaging period. Red arrowheads show the time points for the images in $\boldsymbol{C}$. Scale bar, $10 \mu \mathrm{m}$. $\boldsymbol{E}$, Initial microglial area at $t=0$ of Before and After sessions are similar in Sham mice (13 cells from 8 mice; $\left.p=0.82^{\mathrm{i}}\right)$. Blue lines represent data from individual microglia, and the black line represents averaged data. $\boldsymbol{F}$, Normalized surveillance area curves during the 60 min imaging period before (blue) and after (red) stimulation in the sham (left) and tDCS (right) mice. Data are represented as the mean \pm SEM. G, Normalized surveillance area at $t=40 \mathrm{~min}$ in Before and After sessions in tDCS-treated mice (normalized by surveillance area at $t=0 /$ Before). Red lines represent data from individual microglia, and the black line represents averaged data. $p$ $=0.014^{\mathrm{j}}$, paired $t$ test. $* * p<0.03$.

morphologic dynamics of individual microglia in the cortex of awake mice using a two-photon microscope (Fig. $6 A)$. We used the Iba1-EGFP mouse, in which EGFP is exclusively expressed in microglia (Hirasawa et al., 2005). We confirmed that microglia showed surveillance activities by continual extension and retraction of their processes in all directions (Davalos et al., 2005; Nimmerjahn et al., 2005). For example, the overlay of $60 \mathrm{~min}$ imaging resulted in an extensive coverage of the area within $\sim 60$ $\mu \mathrm{m}$ from the soma, while the soma position remained unmoved (Fig. 6C). We defined normalized surveillance area as the proportion of cumulative microglia-occupied area at a given time relative to a start time (Fig. 6D). To check whether laser scanning has an impact on microglial morphology, we compared the occupied area of each monitored microglia at the beginnings of Before and After imaging sessions of the Sham-treated group (Fig. 6E). We found no significant difference, suggesting that the effect of laser irradiation on microglial morphologic dynamics is negligible.

While the evolution of a normalized surveillance area varied considerably among individual microglia, the average trace converged to a gradually decelerating curve (Fig. 6F). The mean surveillance area after $60 \mathrm{~min}$ did not differ significantly between before and after sham stimulation. Remarkably, the mean surveillance area index curve of tDCS mice (i.e., After session) is plotted lower than the control condition (i.e., Before session). We assessed the surveillance area change of individual microglia by taking the ratio of the surveillance area indices during Before and After sessions, demonstrating a significant decrease of surveillance area by tDCS $(t=40 \mathrm{~min}$, $p=0.014^{j}$, paired $t$ test; Fig. $6 G$ ).

Furthermore, we addressed whether noradrenergic signaling is involved in this tDCS-induced microglial surveillance reduction by prazosin or ICl181551 pretreatment in 

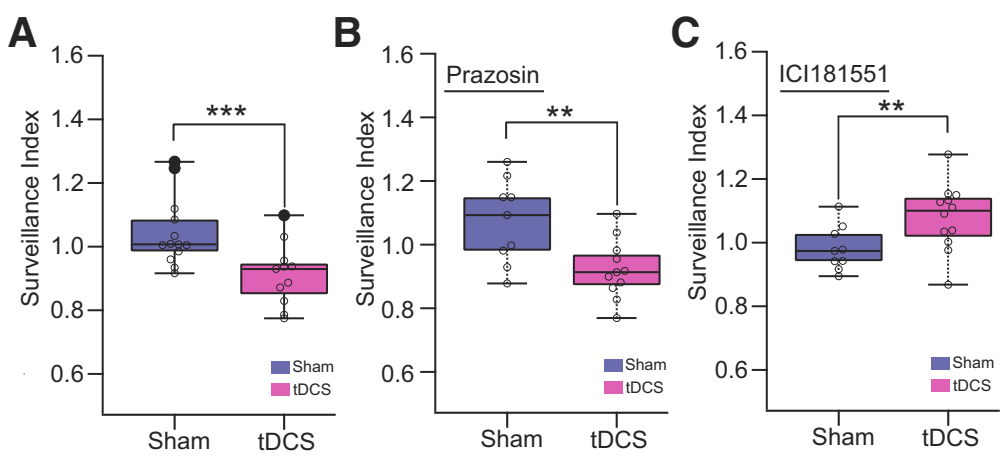

Figure 7. Microglial surveillance is compromised by tDCS. $\boldsymbol{A}$, Surveillance index at $t=40 \mathrm{~min}$ after sham/tDCS treatment in no drug-treated animals (Sham group: 13 cells from 8 mice; tDCS group: 11 cells from 8 mice; $p=0.006^{k}$ ). $\boldsymbol{B}$, Surveillance index comparison in prazosin pretreated mice (Sham group: 9 cells from 2 mice; tDCS group: 11 cells from 3 mice; $p=0.015$ ). $\boldsymbol{C}$, Surveillance index comparison in ICl181551-pretreated mice (Sham group: 9 cells from 3 mice; tDCS group: 12 cells from 3 mice; $p$ $\left.=0.023^{\mathrm{m}}\right)$ Mann-Whitney Wilcoxon rank sum test. $* * p<0.03 ; * * * p<0.01$

awake mice (Fig. 7A). As a reference, we computed the surveillance index comparing Before and After sessions at 40 min after the start of respective sessions. As expected from the previous analysis (Fig. 6G), the surveillance index of tDCS experiments was significantly reduced (Fig. 7A). Prazosin-treated mice showed a similar significant reduction of surveillance index after tDCS (Fig. 7B). By contrast, ICl181551 treatment abolished tDCS-induced reduction of microglia surveillance, and a trend for increased surveillance was apparent (Fig. $7 C$ ). These results point to a significant role of the B2AR in the inhibition of microglial surveillance activity after tDCS.

\section{Discussion}

The present experiments report that tDCS induces subtle, but significant, alterations of Iba1 distribution and microglial motility in the cerebral cortex in awake mice. Furthermore, these alterations were dependent on (nor)adrenergic receptors, which is in line with the results of an earlier study that described tDCS-induced A1ARdependent astrocytic $\mathrm{Ca}^{2+}$ surges (Monai et al., 2016). Notably, while astrocytic $\mathrm{Ca}^{2+}$ responses occur during tDCS, morphologic alterations of microglia occurred after a few hours.

We demonstrated that the microglial soma is enlarged after tDCS. Remarkably, the soma enlargement occurs only in awake mice. It is well established that microglial morphology is radically altered by LPS-induced inflammation (Kondo et al., 2011; Kozlowski and Weimer, 2012; Kongsui et al., 2015). LPS-induced microglial alterations are obvious even with a low dosage of $100 \mu \mathrm{g} / \mathrm{kg}$, whereby $\sim 20 \%$ soma enlargement has been reported in the prefrontal cortex (Kongsui et al., 2015). The tDCSinduced microglial soma enlargement of a mere several percentage points in the current study is relatively modest. Moreover, no obvious change was detected in ramified processes. As general anesthesia compromises astrocytic $\mathrm{Ca}^{2+}$ activation, in particular noradrenergically driven large-scale and synchronized $\mathrm{Ca}^{2+}$ surges (Thrane et al., 2012; Ding et al., 2013), microglial changes by tDCS conceivably depend on the elevated noradrenergic tone during awake states. On the other hand, some studies have reported significant changes in anesthetized mice that underwent tDCS. For instance, one study reported that enhancements of GFAP and brain-derived neurotrophic factor (BDNF) in anesthesia changed gene expression (de Souza Nicolau et al., 2018). Another study showed long-lasting antidepressive behavioral effects (Peanlikhit et al., 2017). However, these studies used stronger stimulation in terms of stimulus current, duration, and/or frequency. Moreover, the anesthesia condition used in the current study is deeper than that in the study by Peanlikhit et al. (2017). Considering the lack of astrocytic $\mathrm{Ca}^{2+}$ surges in this condition (Extended Data Fig. 1-1), our results support the involvement of volumetransmitted neuromodulators in tDCS.

A few studies have examined cortical microglia after tDCS. For instance, Rueger et al. (2012) reported that multisession tDCS of 5-10 d induced a mild sign of microglial activation as observed by an upregulation of Iba1 immunohistochemical signals. The current density used in the study by Rueger et al. (2012) was $\sim 150 \mathrm{~A} / \mathrm{m}^{2}$, whereas that used in the current study is $<30 \mathrm{~A} / \mathrm{m}^{2}$. Considering the study by Gellner et al. (2016), which reported a microglial activation threshold of $30-50 \mathrm{~A} / \mathrm{m}^{2}$ with light isoflurane anesthesia, it is conceivable that our experiments were performed in near-threshold conditions. The tDCS-induced microglial soma enlargement and Iba1 signal intensity distribution shift are different from the microglial morphologic alterations reported in a rodent model of electroconvulsive therapy (ECT), in which obvious reductions in process ramification and Iba1 expression occur (Jinno and Kosaka, 2008). The pronounced alterations of microglia by ECT are most likely caused by the high-intensity electric stimulation that induces seizures. By contrast, cortical neuronal discharge activity remains undisturbed by tDCS (Monai et al., 2016; Vöröslakos et al., 2018).

We find that tDCS-induced soma enlargement is dependent on noradrenergic signaling. Moreover, the prazosin and $\mathrm{IP}_{3} \mathrm{R} 2-\mathrm{KO}$ mouse (which lacks astrocytic $\mathrm{Ca}^{2+}$ surges) experiments suggest a key mechanism linked to A1AR activation. The previous reports of relative abundance of A1ARs in astrocytes over microglia (Hertz et al., 2010; Zhang et al., 2014) and A1AR-dependent tDCS- 
induced astrocytic $\mathrm{Ca}^{2+}$ surges (Monai et al., 2016) support the idea that astrocytic activation exerts effects on microglia. While this is intriguing, neither the prazosin nor the $\mathrm{IP}_{3} \mathrm{R} 2 \mathrm{KO}$ mouse experiment is cell type specific; therefore, it is possible that direct noradrenergic activation of microglia causes soma enlargement. Indeed, B2AR inhibition by ICl181551 also disrupted microglial somatic enlargement. Functional and transcriptomic evidence underwrites the enriched expression of B2ARs in microglia (Tanaka et al., 2002; Gyoneva and Traynelis, 2013; Zhang et al., 2014).

By imaging microglial morphology in awake mice, we found that tDCS attenuates microglial motility. This effect was also dependent on B2ARs, but not on A1ARs. The inhibitory effect of microglial B2ARs on motility is consistent with the in vitro observation by Gyoneva and Traynelis (2013) and recent in vivo observations in awake mice (Liu et al., 2019; Stowell et al., 2019). It is tempting to speculate that the brake on microglial surveillance creates an opportunity for relevant synapses to establish an initial stage of synaptic plasticity. Microglia have been demonstrated to be a source for BDNF (Parkhurst et al., 2013), a pivotal neurotrophin for synaptic plasticity and neurogenesis. Interestingly, tDCS upregulates Bdnf (de Souza Nicolau et al., 2018), promotes BDNF-dependent synaptic plasticity (Fritsch et al., 2010), and causes epigenetic modification to Bdnf genomic regions (Podda et al., 2016). It remains to be shown whether BDNF synthesis is promoted by (nor)adrenergic activation as is reported in astrocytes (Jurič et al., 2008). In addition to astrocyteneuron interactions (Monai and Hirase, 2016; Cocco et al., 2018), our results advocate for the inclusion of microglia as a functional component of the tDCS mechanism via adrenergic receptor activation.

One of the limitations of the current study is the lack of microglia-specific molecular manipulations. While it remains undetermined whether the microglial changes observed in this study have causal roles for positive outcomes of tDCS, several groups have consistently reported inflammation-associated microglial soma enlargement (Chen et al., 2012; Kozlowski et al., 2012; Kongsui et al., 2015). Brain inflammation activates microglia and leads to the production of proinflammatory molecules such as TNF- $\alpha, \mathrm{IL}-1 \beta$, and IL-6 (Hanisch, 2002). It is possible that these cytokines are involved in the synaptic plasticity induced by tDCS. For instance, it has been demonstrated that the glial TNF- $\alpha$ has a pivotal role in the regulation of homeostatic synaptic plasticity (Stellwagen and Malenka, 2006). Future studies should address the causal relationship, for instance by microglial B2AR knock-out mice combined with tDCS and behavioral performance.

\section{References}

Araque A, Carmignoto G, Haydon PG, Oliet SHR, Robitaille R, Volterra A (2014) Gliotransmitters travel in time and space. Neuron 81:728-739.

Bekar LK, He W, Nedergaard M (2008) Locus coeruleus $\alpha$-adrenergic-mediated activation of cortical astrocytes in vivo. Cereb Cortex 18:2789-2795.
Bikson M, Grossman P, Thomas C, Zannou AL, Jiang J, Adnan T, Mourdoukoutas AP, Kronberg G, Truong D, Boggio P, Brunoni AR, Charvet L, Fregni F, Fritsch B, Gillick B, Hamilton RH, Hampstead BM, Jankord R, Kirton A, Knotkova H, et al. (2016) Safety of transcranial direct current stimulation: evidence based update 2016. Brain Stimul 9:641-661.

Brunoni AR, Nitsche MA, Bolognini N, Bikson M, Wagner T, Merabet L, Edwards DJ, Valero-Cabre A, Rotenberg A, Pascual-Leone A, Ferrucci R, Priori A, Boggio PS, Fregni F (2012) Clinical research with transcranial direct current stimulation (tDCS): challenges and future directions. Brain Stimul 5:175-195.

Chen Z, Jalabi W, Shpargel KB, Farabaugh KT, Dutta R, Yin X, Kidd GJ, Bergmann CC, Stohlman SA, Trapp BD (2012) Lipopolysaccharide-induced microglial activation and neuroprotection against experimental brain injury is independent of hematogenous TLR4. J Neurosci 32:11706-15.

Cocco S, Podda MV, Grassi C (2018) Role of BDNF signaling in memory enhancement induced by transcranial direct current stimulation. Front Neurosci 12:427.

Davalos D, Grutzendler J, Yang G, Kim JV, Zuo Y, Jung S, Littman DR, Dustin ML, Gan W-B (2005) ATP mediates rapid microglial response to local brain injury in vivo. Nat Neurosci 8:752-758.

de Souza Nicolau E, de Alvarenga KAF, Tenza-Ferrer H, Nogueira MCA, Rezende FD, Nicolau NF, Collodetti M, de Miranda DM, Magno LAV, Romano-Silva MA (2018) Transcranial direct current stimulation (tDCS) in mice. J Vis Exp (139):e58517.

Dedoncker J, Brunoni AR, Baeken C, Vanderhasselt M-A (2016) A systematic review and meta-analysis of the effects of transcranial direct current stimulation (tDCS) over the dorsolateral prefrontal cortex in healthy and neuropsychiatric samples: influence of stimulation parameters. Brain Stimul 9:501-517.

Ding F, O'Donnell J, Thrane AS, Zeppenfeld D, Kang H, Xie L, Wang F, Nedergaard M (2013) $\alpha 1$-Adrenergic receptors mediate coordinated $\mathrm{Ca} 2+$ signaling of cortical astrocytes in awake, behaving mice. Cell Calcium 54:387-394.

Fregni F, Pascual-Leone A (2007) Technology insight: noninvasive brain stimulation in neurology-perspectives on the therapeutic potential of rTMS and tDCS. Nat Clin Pract Neurol 3:383-393.

Fritsch B, Reis J, Martinowich K, Schambra HM, Ji Y, Cohen LG, Lu B (2010) Direct current stimulation promotes BDNF-dependent synaptic plasticity: potential implications for motor learning. Neuron 66:198-204.

Futatsugi A, Nakamura T, Yamada MK, Ebisui E, Nakamura K, Uchida K, Kitaguchi T, Takahashi-Iwanaga H, Noda T, Aruga J, Mikoshiba K (2005) IP3 receptor types 2 and 3 mediate exocrine secretion underlying energy metabolism. Science 309:2232-2234.

Gellner A-K, Reis J, Fritsch B (2016) Glia: a neglected player in non-invasive direct current brain stimulation. Front Cell Neurosci 10:188.

Gyoneva S, Traynelis SF (2013) Norepinephrine modulates the motility of resting and activated microglia via different adrenergic receptors. J Biol Chem 288:15291-15302.

Hanisch UK (2002) Microglia as a source and target of cytokines. Glia 40:140-155.

Hertz L, Lovatt D, Goldman SA, Nedergaard M (2010) Adrenoceptors in brain: cellular gene expression and effects on astrocytic metabolism and $[\mathrm{Ca}(2+)]$ i. Neurochem Int 57:411-420.

Hines DJ, Hines RM, Mulligan SJ, Macvicar BA (2009) Microglia processes block the spread of damage in the brain and require functional chloride channels. Glia 57:1610-1618.

Hirasawa T, Ohsawa K, Imai Y, Ondo Y, Akazawa C, Uchino S, Kohsaka S (2005) Visualization of microglia in living tissues using Iba1-EGFP transgenic mice. J Neurosci Res 81:357-362.

Horvath JC, Forte JD, Carter O (2015a) Evidence that transcranial direct current stimulation (tDCS) generates little-to-no reliable neurophysiologic effect beyond MEP amplitude modulation in healthy human subjects: a systematic review. Neuropsychologia 66:213236.

Horvath JC, Forte JD, Carter O (2015b) Quantitative review finds no evidence of cognitive effects in healthy populations from single- 
session transcranial direct current stimulation (tDCS). Brain Stimul 8:535-550.

Jalali R, Miall RC, Galea JM (2017) No consistent effect of cerebellar transcranial direct current stimulation on visuomotor adaptation. $J$ Neurophysiol 118:655-665.

Jinno S, Kosaka T (2008) Reduction of Iba1-expressing microglial process density in the hippocampus following electroconvulsive shock. Exp Neurol 212:440-447.

Jurič DM, Lončar D, C`arman-Kržan M (2008) Noradrenergic stimulation of BDNF synthesis in astrocytes: mediation via $\alpha 1$ - and $\beta 1 / \beta 2$-adrenergic receptors. Neurochem Int 52:297-306.

Kondo S, Kohsaka S, Okabe S (2011) Long-term changes of spine dynamics and microglia after transient peripheral immune response triggered by LPS in vivo. Mol Brain 4:27.

Kongsui R, Johnson SJJ, Graham BAA, Nilsson M, Walker FRR (2015) A combined cumulative threshold spectra and digital reconstruction analysis reveal structural alterations of microglia within the prefrontal cortex following low-dose LPS administration. Neuroscience 310:629-640.

Kozlowski C, Weimer RM (2012) An automated method to quantify microglia morphology and application to monitor activation state longitudinally in vivo. PLoS One 7:e31814.

Kunzelmann K, Meier L, Grieder M, Morishima Y, Dierks T (2018) No effect of transcranial direct current stimulation of the auditory cortex on auditory-evoked potentials. Front Neurosci 12:880.

Kuo H-I, Paulus W, Batsikadze G, Jamil A, Kuo M-F, Nitsche MA (2017) Acute and chronic effects of noradrenergic enhancement on transcranial direct current stimulation-induced neuroplasticity in humans. J Physiol 595:1305-1314.

Liu Y, Li Y, Eyo UB, Chen T, Umpierre A, Zhu J, Bosco DB, Dong H, Wu L-J (2019) Neuronal network activity controls microglial process surveillance in awake mice via norepinephrine signaling. bioRxiv. Advance online publication. Retrieved August 24, 2019. doi:10.1101/557686.

Medina J, Cason S (2017) No evidential value in samples of transcranial direct current stimulation (tDCS) studies of cognition and working memory in healthy populations. Cortex 94:131-141.

Monai H, Hirase H (2016) Astrocytic calcium activation in a mouse model of tDCS—extended discussion. Neurogenesis 3:e1240055.

Monai H, Hirase H (2018) Astrocytes as a target of transcranial direct current stimulation (tDCS) to treat depression. Neurosci Res 126: 15-21.

Monai H, Ohkura M, Tanaka M, Oe Y, Konno A, Hirai H, Mikoshiba K, Itohara S, Nakai J, Iwai Y, Hirase H (2016) Calcium imaging reveals glial involvement in transcranial direct current stimulation-induced plasticity in mouse brain. Nat Commun 7:11100.

Nimmerjahn A, Kirchhoff F, Helmchen F (2005) Resting microglial cells are highly dynamic surveillants of brain parenchyma in vivo. Science 308:1314-1318.

Nitsche MA, Cohen LG, Wassermann EM, Priori A, Lang N, Antal A, Paulus W, Hummel F, Boggio PS, Fregni F, Pascual-Leone A (2008) Transcranial direct current stimulation: state of the art 2008. Brain Stimul 1:206-223.

Nitsche MA, Boggio PS, Fregni F, Pascual-Leone A (2009) Treatment of depression with transcranial direct current stimulation (tDCS): a review. Exp Neurol 219:14-19.

Parkhurst CN, Yang G, Ninan I, Savas JN, Yates JR, Lafaille JJ, Hempstead BL, Littman DR, Gan W-B (2013) Microglia promote learning-dependent synapse formation through brain-derived neurotrophic factor. Cell 155:1596-1609.

Peanlikhit T, Van Waes V, Pedron S, Risold P-Y, Haffen E, Etiévant A, Monnin J (2017) The antidepressant-like effect of tDCS in mice: a behavioral and neurobiological characterization. Brain Stimul 10: 748-756.

Pfeiffer T, Avignone E, Nägerl UV (2016) Induction of hippocampal long-term potentiation increases the morphological dynamics of microglial processes and prolongs their contacts with dendritic spines. Sci Rep 6:32422.

Podda MV, Cocco S, Mastrodonato A, Fusco S, Leone L, Barbati SA, Colussi C, Ripoli C, Grassi C (2016) Anodal transcranial direct current stimulation boosts synaptic plasticity and memory in mice via epigenetic regulation of Bdnf expression. Sci Rep 6:22180.

Rueger MA, Keuters MH, Walberer M, Braun R, Klein R, Sparing R, Fink GR, Graf R, Schroeter M (2012) Multi-session transcranial direct current stimulation (tDCS) elicits inflammatory and regenerative processes in the rat brain. PLoS One 7:e43776.

Souza A, Martins DF, Medeiros LF, Nucci-Martins C, Martins TC, Siteneski A, Caumo W, dos Santos ARS, Torres ILS (2018) Neurobiological mechanisms of antiallodynic effect of transcranial direct current stimulation (tDCS) in a mice model of neuropathic pain. Brain Res 1682:14-23.

Stellwagen D, Malenka RC (2006) Synaptic scaling mediated by glial TNF- $\alpha$. Nature 440:1054-1059.

Stowell RD, Grayson OS, Ryan PD, Hanna NB, Lordy AK, Jean MB, Edward B, Mriganka S, Ania KM (2019) Noradrenergic signaling in wakeful states inhibits microglial surveillance and synaptic plasticity in the mouse visual cortex. bioRxiv. Advance online publication. Retrieved August 24, 2019. doi:10.1101/556480.

Tanaka KF, Kashima H, Suzuki H, Ono K, Sawada M (2002) Existence of functional beta1- and beta2-adrenergic receptors on microglia. J Neurosci Res 70:232-237.

Thrane AS, Thrane VR, Zeppenfeld D, Lou N, Xu Q, Nagelhus EA, Nedergaard M, Rangroo Thrane V (2012) General anesthesia selectively disrupts astrocyte calcium signaling in the awake mouse cortex. Proc Natl Acad Sci U S A 109:18974-18979.

Turkakin E, Akbıyık S, Akyol B, Gürdere C, Çakmak YÖ, Balcı F (2018) Differential bilateral primary motor cortex tDCS fails to modulate choice bias and readiness in perceptual decision making. Front Neurosci 12:410.

Vöröslakos $M$, Takeuchi $Y$, Brinyiczki $K$, Zombori T, Oliva A, Fernández-Ruiz A, Kozák G, Kincses ZT, Iványi B, Buzsáki G, Berényi A (2018) Direct effects of transcranial electric stimulation on brain circuits in rats and humans. Nat Commun 9:483.

Wake H, Moorhouse AJ, Jinno S, Kohsaka S, Nabekura J (2009) Resting microglia directly monitor the functional state of synapses in vivo and determine the fate of ischemic terminals. J Neurosci 29:3974-80.

Wake H, Moorhouse AJ, Miyamoto A, Nabekura J (2013) Microglia: actively surveying and shaping neuronal circuit structure and function. Trends Neurosci 36:209-217.

Zhang Y, Chen K, Sloan SA, Bennett ML, Scholze AR, O'Keeffe S, Phatnani HP, Guarnieri P, Caneda C, Ruderisch N, Deng S, Liddelow SA, Zhang C, Daneman R, Maniatis T, Barres BA, Wu JQ (2014) An RNA-sequencing transcriptome and splicing database of glia, neurons, and vascular cells of the cerebral cortex. J Neurosci 34:11929-11947. 